\title{
Dietary patterns in Europe: the Food4Me proof of principle study
}

\author{
C. Woolhead ${ }^{1}$, M.C. Walsh ${ }^{1}$, M.J. Gibney ${ }^{1}$, H. Daniel ${ }^{2}$, C.A. Drevon ${ }^{3}$, J.A. Lovegrove ${ }^{4}$, \\ Y. Manios ${ }^{5}$, J.A. Martinez ${ }^{6}$, JC Mathers ${ }^{7}$, I. Traczyk ${ }^{8}$, W.H.M Saris ${ }^{9}$, ER Gibney ${ }^{1}$ and \\ L. Brennan ${ }^{1}$ on behalf of the Food4Me study \\ ${ }^{1}$ Institute of Food and Health, University College Dublin, Ireland, ${ }^{2}$ ZIELResearch Center of Nutrition and Food \\ Sciences Technische Universität München, Germany, ${ }^{3}$ Department of Nutrition, University of Oslo, Norway, ${ }^{4} \mathrm{Hugh}$ \\ Sinclair Unit of Human Nutrition and Institute for Cardiovascular and Metabolic Research, University of Reading, \\ RG6 6AP UK, ${ }^{5}$ Department of Nutrition and Dietetics, Harokopio University of Athens, Athens, Greece, ${ }^{6}$ University of \\ Navarra, Pamplona, Spain, ${ }^{7}$ Human Nutrition Research Centre, Newcastle University, NE4 5PL, UK, ${ }^{8}$ National Food \\ \& Nutrition Institute, Warsaw, Poland and ${ }^{9}$ Department of Human Biology, NUTRIM, Maastricht University, \\ Maastricht, The Netherlands
}

This abstract was awarded an oral presentation prize sponsored by Sugar Nutrition UK and the Food Safety Authority of Ireland.

Dietary pattern analysis aims to identify overall dietary intake patterns in populations. Previous studies have examined dietary patterns across populations in order to examine possible geographical nuances in terms of food intake ${ }^{(1,2)}$. A useful method to determine dietary patterns is clustering which classifies individuals into groups based on similarity of overall intake. These patterns could be translated into public health guidelines or used in a personalised nutrition (PN) setting ${ }^{(3)}$. The aim of the present study was to identify dietary patterns across Europe using dietary data from Food4Me proof of principle (PoP) study.

The Food4Me study was a web-based, randomized control trial comparing the effects of different levels of PN on health outcomes, conducted over 6 months in 7 different countries: Ireland, UK, Spain, Greece, Germany, Netherlands and Poland ${ }^{(4)}$. The Food4Me online food frequency questionnaire was used to collect dietary intake data on 162 food items ${ }^{(5)}$. Reported intakes of food items were reduced into food groups and percentage contribution of each food group to energy intake (\% TE) was calculated. Dietary patterns were derived using two-step and k-means clustering with food group intake values (\% TE). General linear models were used to compare $\%$ TE within each food group between clusters within each country, adjusted for age, gender and BMI.

Baseline data from a total of 1212 participants was used in this study. Food items were reduced into 33 food groups based on nutritional similarity. Either two or three clusters were identified within each country with the two main dietary patterns named as "Prudent" and "Western/processed". These were based on comparisons of mean food group intakes (\% TE) between clusters (P< 0.05). Across all countries, the "Prudent" diet was highest in plant-based foods, including fruit intake, in the range of 6.14-10.67 \%TE. Within this pattern, fish was consumed in high amounts in Germany, Ireland and Poland and UK, and dairy was consumed in high amounts in Germany, Ireland, Poland and Greece. The Prudent diet of the Netherlands was characterized by plant-based foods only. The Prudent diet of participants in Spain, UK and Poland all consumed high amounts of low fat spreads/oils. The "Western/processed" diet was high in energy-dense or processed foods such as meat products or chips/processed potatoes. Participants in the "Western/processed" diet group also consumed high-energy beverages (Germany, Ireland, Netherlands and Poland), high-carbohydrate foods such as white bread (Greece, Poland and UK), savouries (Germany, Greece, Ireland, Netherlands, Spain) and high-fat foods such as biscuits/cakes (Poland, Greece, UK). German participants in this group also had high intakes of alcohol (4.63 $\pm 4.45 \%$ TE). Other dietary patterns included a "Low fat dairy" diet (Ireland, Spain and UK), and a "Confectionary" diet (Germany).

The present dietary pattern analysis has identified distinct patterns that are country specific. Distinct "Prudent" and "Western/processed" patterns were established in each country, but the main contributing food groups were country-dependent. The "Low fat dairy" pattern was unique to three countries. Future work will be directed towards understanding if these patterns have an impact on metabolic health.

This work was funded by Food4me (KBBE.2010·2·3-02, Project no.265494), http://www.food4me.org/.

1. Bamia C, Orfanos P, Ferrari P et al. (2005) Br J Nutr. 94(01), 100-13.

2. Baxter AJ, Coyne T'\& McClintock C. (2006) Asia Pac J Clin Nutr. 15(2).

3. Gibney MJ, \& Walsh MC. (2013) Proc Nutr Soc. 72(2), 219-25.

4. Celis-Morales C, Livingstone KM, Marsaux CF et al. (2015) Genes Nutr. 10(1), 450.

5. Forster H, Fallaize R, Gallagher C et al. (2014) J Med Internet Res.; 16(6). 